\begin{tabular}{ccc}
\hline & International Journal of Engineering \& Technology, $7(3.13)(2018)$ 176-182 \\
SPC & International Journal of Engineering \& Technology \\
Website: $w$ ww.sciencepubco.com/index.php/IJET & Research paper \\
\hline
\end{tabular}

\title{
Nonlinear effects in WDM Networks
}

\author{
Kenza GAIZI $^{12 *}$, Pr. Dr. Fouad Mohammed ABBOU ${ }^{1}$, Pr. Dr. Farid ABDI ${ }^{2}$ \\ ${ }^{1}$ School of Science and Engineering, Al Akhawayn University Ifrane, Morocco \\ ${ }^{2}$ Laboratoire de Signaux, Systèmes et Composants, Université Sidi Mohammed Ben Abdellah, Faculté des Sciences et Techniques, \\ Fès, Morocco \\ *Corresponding author E-mail: kenza.gaizi@usmba.ac.ma
}

\begin{abstract}
This paper studies nonlinear effects in WDM Network. The focus is on both the propagation of a unique signal as well as a set of them in optical fibers in a WDM network. The paper presents an analytical model to study the effects of having nonlinearities in a WDM system. Three main nonlinear effects are studied here are Cross Phase Modulation, Self-Phase Modulation, and Four Wave Mixing. Simulations are performed on up to 265 channels WDM System.
\end{abstract}

Keywords: WDM network; nonlinear effects; Four Wave Mixing; Cross Phase Modulation.

\section{Introduction}

Before diving into the subject, it is important to distinguish between two types of Fiber Optics: (1) Linear and (2) Nonlinear. The Linearity of the first category comes from the fact that the medium properties of the fiber do not depend on the signal. On the other hand, when the material properties of the fiber get affected by the signal itself we would then be in the second category of Nonlinear Fiber Optics. In this paper, we will be discussing the propagation of signal in the fiber taking into account nonlinear effects. We would need to answer the following question: If the medium properties change in a fiber due to nonlinear effects, what are the implications on the signal?

\section{Signal Propagation within Optical Fibre}

\subsection{Propagation of One Signal within the Optical Fibre}

The 80 s was marked as an era during which many researchers were invested in experimental work to establish the equivalence of light and electromagnetic propagation [1]. Marking the beginning of radio telecommunications, in 1887, Heinrich Hertz was able to generate and detect radio waves in the laboratory [2]. This came validating Maxwell's electromagnetic theory [3], according to which, electromagnetic waves could be emitted from an oscillating electric dipole, propagating via space at the speed of light; while exhibiting wave-like characteristics of light propagation [1], [2]. Let's take an example of a pulse that we need to transmit on the optical fibre in the linear domain. The properties of the propagation of such pulse are only decided by the medium properties [4] [5]. We could simply then solve the wave equation without worrying about the amplitude of the pulse, and we can get the normal distribution, propagation constant and find out about the propagation characteristics of a path. Now imagine that the medium properties are modified by the pulse itself. Let's say that the refractive index of the optical fibre is related to the intensity of light. Now, let's assume that the refractive index increases with the intensity of light. This means that the whole pulse is actually not going to see the same propagation parameters. That is to say, at the centre of the pulse when the light is intense, the refractive index seen by the pulse is different than on the edge of the pulse when the light is less intense. Because of that, the refractive index on the edge is less than the refractive index seen by the pulse in its centre. If we try to send a continuous signal in the optical fibre, any small perturbation will break the signal into pulses. This is phenomenon is referred to, in the fields of nonlinear optics and fluid dynamics, as modulation instability. What happens here is that the deviations from a periodic waveform are reinforced by nonlinearities, generating by that spectral sidebands while also causing a breakup of the waveform into a set of pulses (wave trains) [6], [7], [8]. Given this, it's important to remember that without taking into consideration the pulse nature of the signal, the nonlinear propagation will not be very effective.

In this paper, I will present a simple formulation and we'll answer the question why is nonlinear fibre optics important. We're also going to investigate if it is important to include the effects of nonlinearity into fibre optics communications or not. If the answer is yes, then, we're going to study if we can we make use of these nonlinear effects to improve the signal propagation from the optical communication point of view?

\subsubsection{Analytical Model}

We're going to begin with taking a very basic model. When the electric field is imposed on a dielectric material, the result is a tiny, compared to the scale of atom dimensions, displacement of both positive and negative charge. Every molecule of the material is characterized by an induced dipole moment and the material is 'polarized' [9]. The induced polarization in the material given by susceptibility of the medium. Normally, we consider only the first order susceptibility due to the dielectric material. However, that's just an approximation. In real life, when light intensity is high, the first order term is simply not adequate and one needs to consider higher order terms into the polarization of the material to account for nonlinear effects. We consider a medium nonlinear, if there's a 
nonlinear relation between the induced polarization $\mathrm{P}$ and the applied electric field E [10]. In general, the induced polarization in the material is $P$, given as:

$$
P=\varepsilon_{0}\left\{\chi^{(1)} \cdot \bar{E}+\chi^{(2)}: \bar{E} \bar{E}+\chi^{(3)}: \bar{E} \bar{E} \bar{E}+\cdots\right\}
$$

$\chi^{(1)}$ is the first order susceptibility, $\chi^{(2)}$ is the second order susceptibility, $\chi^{(3)}$ is the third order susceptibility, and so on. $\mathrm{E}$ is the imposed electric field [11]. The dominant term from the Induced Polarization equation is $\chi^{(1)}$ and it contributes to dielectric constant, the second term $\chi^{(2)}$ is small for glass $\left(\mathrm{S}_{\mathrm{i}} \mathrm{O}_{2}\right)$, and the third term $\chi^{(3)}$, also known as the third order susceptibility says that the refractive index is proportional to the square of the electrical field, and that's the one that'll contribute in glass to the nonlinear effects. Notice here that when the intensity is very low, the second and third terms are negligible [12].

$$
\bar{n}\left(\omega,|E|^{2}\right)=\bar{n}(\omega)+n_{2}|E|^{2}
$$

$\mathrm{n}_{2}$ is the nonlinearity coefficient (a.k.a Kerr nonlinearity) [13]. It is material dependent and also dependent on the third order susceptibility of the medium as shown below:

$$
n_{2}=\left(\frac{3}{8} n\right) \chi^{(3)} \approx 2.3 \times 10^{-22} m^{2} / v^{2}(\text { for glass })
$$

Comparing the nonlinear coefficient of glass to other materials we can see that the glass's coefficient is 2 orders of magnitude smaller. Now if this is the case for glass compared to other bulk materials, should we really care about the effects of nonlinearities in optical networks at all? To properly answer this question, we need to understand how do nonlinearities manifest in optical fibres compared to the bulk materials and if there's situational difference.

\subsubsection{Nonlinearities in Bulk Material}

Let's start with the bulk material. When the light beam enters the bulk material, the nonlinear interaction will be in the light and the matter of that bulk material. On the other hand, when the light enters the fibre, it travels a long distance during which it keeps interacting with the fibre, resulting in a cumulative nonlinear effect. To compare between the bulk and the fibre material, one can check how much enhancement of nonlinear interaction takes place in fibre material compared to a bulk material. Let's now define a Figure of merit or efficiency parameter for nonlinear interaction, say where:

$$
\eta \sim I \times L_{e f f}
$$

I is the intensity of light, which is the power intensity per meter area $\left(\mathrm{w} / \mathrm{m}^{2}\right), \mathrm{L}_{\text {eff }}$ is the interaction length in (m) [12]. Equation (4) shows that increasing efficiency of nonlinear interaction can be achieved by either increasing the intensity of light, the interaction length, or both if that's possible.

Let's say, we would like to increase the light intensity in some bulk material. To do that one would need to focus the light beam using a lens. So, by focusing of the light, the spot size becomes smaller and smaller and then the light intensity can be enhanced. If we have a Gaussian Light Beam, with a spot radius of $\omega_{0}$ radius, or a diameter of $2 \omega_{0}$. We can represent the Intensity of Light I as the optical beam power divided by the area of the spot as per the equation:

$$
I=\frac{P}{\pi \omega_{0}^{2}}
$$

Where $\mathrm{P}$ is the light beam power and $\omega 0$ is the light spot radius in the bulk material from this equation, we can see that the length over which the beam focus is related to the size of the spot. So, in a bulk medium, when we try to tightly focus the optical beam, the interaction length gets affected as well [12]. The Interaction length in bulk optics is:

$$
L_{e f f}=\frac{\pi \omega_{0}^{2}}{\lambda}
$$

Let's now compute the figure of merit:

$$
\eta_{\text {bulk }}=I \times L_{e f f}=\frac{P}{\pi \omega_{0}^{2}} \times \frac{\pi \omega_{0}^{2}}{\lambda}=\frac{P}{\lambda}
$$

This shows the figure of merit, or the interaction efficiency is independent of the size of the focusing beam. So, in bulk material, if we want to enhance the effect of nonlinearity, and want to do a certain wavelength, the only option we have is to increase the optical power. So for research focusing on bulk material nonlinearities, high power is required so that nonlinearities can start getting induced effectively and allow researchers to study their effects.

\subsubsection{Nonlinearities in Bulk Material}

Now, let's compare the situation with Optical Fibre. In Optical fibre, when the light gets in, it remains focused, or confined to a region which is the size of the core. Since the loss on the optical fibre is very small, the intensity reduces but very slowly, allowing it to remain almost constant over some tens of kilometres. In the fibre case, let's define the radius of the optical fibre core as a [12]. Hence, the light intensity I would be defined as:

$$
I=\frac{P}{\pi a^{2}}
$$

When the light propagates through the optical fibre, it exponentially decreases. Let's define the power attenuation constant as $\alpha$ and the power function as per [12] [14] below:

$$
P(z)=P(0) e^{-\alpha z}
$$

Now, if we take a distance $z_{0}=\frac{1}{\alpha}$, we will have:

$$
P\left(z_{0}\right)=P(0) e^{-\alpha z}=P(0) e^{-\alpha \frac{1}{\alpha}}=P(0) e^{-1}
$$

We can then say, in the case of optical fibre, that $\mathrm{L}_{\text {eff }} \approx 1 / \alpha$. Now, let's now compute the figure of merit for the optical fibre:

$$
\eta_{f i b e r}=I \times L_{e f f}=\frac{P}{\pi \alpha^{2}} \times \frac{1}{\alpha}
$$

\subsubsection{Nonlinearities in Bulk Material and Optical Fiber}

We can find how much interaction efficiencies increase in the optical fibre compared to bulk material, we can find out by taking the ratio of $\eta_{\text {bulk }}$ and $\eta_{\text {fiber }}$ as shown below:

$$
\frac{\eta_{\text {fiber }}}{\eta_{\text {bulk }}}=\frac{\frac{P}{\pi \alpha^{2}} \times \frac{1}{\alpha}}{\frac{P}{\lambda}}=\frac{\lambda}{\pi a^{2} \alpha}
$$

Let's put some parameters for a typical optical fibre: 


$$
\begin{aligned}
& a \sim 2 \mu \mathrm{m} \\
& \alpha=0.2 \mathrm{~dB} \mathrm{~km}^{-1}=0.2 \times 10^{-3} \mathrm{~dB} \mathrm{~m}^{-1} \simeq 8 \times 10^{-5} \mathrm{~m}^{-1} \\
& \lambda \sim 1550 \mathrm{~nm}=1.55 \mu \mathrm{m} \\
& \qquad \frac{\eta_{\text {fiber }}}{\eta_{\text {bulk }}}=\frac{\lambda}{\pi a^{2} \alpha} \simeq 10^{9}
\end{aligned}
$$

So for the same parameters, the nonlinear effects inside optical fibres is enhanced by 1 billion times than those inside of the bulk material. This also means that whatever affects you can see in bulk material with 1 Watt of power, the same effect inside optical fibre can be seen with only 1 nano Watt of power. This means that with the power which we deal with in Optical Fibre in the order of microWatt or milliWatt, the nonlinear effects will certainly be present because once the power will be confined in optical fibre, it will keep interacting with the fibre over long kilometres.

Comparing the Light intensity required to get nonlinear effects in Fibre v.s. in bulk material. We see that in Fibre, such Intensity is at least two orders of magnitude smaller. Now, in fibre optic communications, we aren't working with single fibres, but rather with some complex systems of multi fibre optics such as WDM and DWDM. Assuming we have an intensity of 1 microWatt per meter square induced per fibre. In a 100 channels WDM System that'll already be 100 microWatt/ meter square. WDM systems are using currently way more channels that this example, and the power intensity inside optical fibre is certainly going to be large enough to really worry about the nonlinear effects in these system while giving us great motivation to study them and analyse ways to make the best use out of them to rather make improvements to the system.

\subsubsection{Light Propagation in Optical Fiber with Nonlinearities}

To analyse the propagation of light in the optical fibre in the presence of nonlinearity, we'll start from the very basic Maxwell's Equations [3] below:

$$
\nabla \times \bar{E}=-\mu_{0} \frac{\delta \bar{H}}{\delta t}
$$

This is assuming that permeability is not a function of time. Also since we're dealing with dielectric material, we can assume that the permeability is the same as that of free space, hence $\mu 0$.

$$
\nabla \times \bar{H}=\frac{\delta \bar{D}}{\delta t}
$$

$\bar{J} \equiv 0$ Assuming the conduction $\mathrm{J}$ of the medium is 0

$$
\nabla \cdot \bar{D}=0
$$

$\delta \equiv 0$ and $\bar{D}$ is the displacement.

$$
\begin{gathered}
\nabla \cdot \bar{B}=0 \\
\bar{D}=\epsilon_{0} \cdot \bar{E}+\bar{P}
\end{gathered}
$$

And since:

$$
P=\epsilon_{0}\left\{\chi^{(1)} \cdot \bar{E}+\chi^{(2)}: \bar{E} \bar{E}+\chi^{(3)}: \bar{E} \bar{E} \bar{E}+\ldots\right\}
$$

Then:

$$
\bar{D}=\varepsilon_{0} \bar{E}+\varepsilon_{0} \cdot \chi^{(1)} E
$$

In normal situations, neglecting the other terms:

$$
\bar{D}=\varepsilon_{0}\left\{1 \chi^{(1)}\right\} \bar{E} \leftarrow \text { Linear case. }
$$

The quantity: $\left\{1+\chi^{(1)}\right\}$ is the dielectric constant of the medium. In general such quantity is complex especially if there are losses in the medium.

$$
\begin{aligned}
& \nabla \times \nabla \times \bar{E}=-\nabla \times\left\{\mu_{0} \frac{\delta \bar{H}}{\delta t}\right\} \\
&=-\mu_{0} \frac{\delta}{\delta t}\{\nabla \times \bar{H}\} \\
&=-\mu_{0} \frac{\delta}{\delta t}\left\{\frac{\delta \bar{D}}{\delta t}\right\} \\
&=-\mu_{0} \frac{\delta^{2}}{\delta t^{2}}\left\{\varepsilon_{0} \bar{E}+\bar{P}\right\} \\
&=-\mu_{0} \frac{\delta^{2}}{\delta t^{2}}\left\{\varepsilon_{0} \bar{E}\right\}-\mu_{0} \frac{\delta^{2} \bar{P}}{\delta t^{2}} \\
& P=\varepsilon_{0}\left\{\chi^{(1)} \cdot \bar{E}+\chi^{(2)}: \bar{E} \bar{E}+\chi^{(3)}: \bar{E} \bar{E} \bar{E}+\ldots\right\}
\end{aligned}
$$

Where $\mu_{0}\left\{\chi^{(1)}, \bar{E}\right\}$ is the Linear Polarization $P_{L}$. While $\mu_{0}\left\{\chi^{(2)}: \bar{E} \bar{E}+\chi^{(3)}: \bar{E} \bar{E} \bar{E}+\ldots\right\}$ is the Nonlinear Polarization $P_{N}$.

$\nabla(\nabla \cdot \bar{E})=-\nabla^{2} \bar{E}=-\frac{1}{c^{2}} \frac{\delta^{2} E}{\delta t^{2}}-\mu_{0}\left\{\frac{\delta^{2} P_{L}}{\delta t^{2}}+\frac{\delta^{2} P_{N}}{\delta t^{2}}\right\}=0$

The wave equation:

$$
\nabla^{2} \bar{E}=-\frac{1}{c^{2}} \frac{\delta^{2} \bar{E}}{\delta t^{2}}=\mu_{0} \frac{\delta^{2} P}{\delta t^{2}}+\mu_{0} \frac{\delta^{2} P_{N}}{\delta t^{2}}
$$

$\bar{E}=E_{0} e^{j \omega_{0} t}$ with $\omega_{0}$ is the signal frequency. $\bar{E}$ is a function of space (r) and time (t).

$$
\hat{E}\left(\bar{r}, \omega-\omega_{0}\right)=\int_{-\infty}^{\infty} E(\bar{r}, t) e^{-j\left(\omega-\omega_{0}\right) t} d t
$$

That is Fourier Transform, spectrum of the electric field as a function of distance:

$$
\nabla^{2} \hat{E}+\varepsilon(\omega) K_{0}^{2} \hat{E}=0
$$

$\varepsilon(\omega)$ is given as:

$$
\begin{gathered}
\varepsilon(\omega)=1+\chi^{(1)}(\omega)+\varepsilon_{N L} \\
\hat{E}\left(\bar{r}, \omega-\omega_{0}\right)=F(\delta, \varphi) \hat{A}\left(z, \omega-\omega_{0}\right) e^{-j \beta_{0} z}
\end{gathered}
$$

$$
\nabla^{2} \perp \bar{F}+\left\{\varepsilon(\omega) K_{0}^{2}-\beta^{2}\right\} F=0
$$

$$
-2 j \beta_{0} \frac{\delta \hat{A}}{\delta z}+\left(\hat{\beta}^{2}-\beta_{0}^{2}\right) \hat{A}=0 \text { with } \frac{\delta^{2} \hat{A}}{\delta z^{2}} \text { negligible }
$$

\subsubsection{Summary}

To summarize, when the light is intense, the induced polarization has high order susceptibility terms also and they cannot be neglected [15]. For a material like glass, the second order susceptibility contribution is negligible because $\mathrm{SiO} 2$ molecule is symmetric, so third order susceptibility term is what contributes to the nonlinear effects. Even if the glass is not a very good nonlinear material because its nonlinear coefficient is two orders of magnitude smaller compared to many well-known nonlinear materials, since the interaction length inside the optical fibre is very large, 
the nonlinear effects are very pronounced inside the optical fibre. Compared to the bulk material, the interaction efficiency in optical fibre is a billion time more so this is a strong case to investigate the nonlinear effects inside the optical fibre. We then started with the Maxwell's equations [3] and wrote down the wave equation in terms of the polarization which includes the nonlinear terms.

\subsection{Propagation of Multiple Signals within the Optical Fibre}

In the previous section, we've studied what happens inside of the optical fibre if there's one signal propagating. In the section below, we'll see what happens inside the optical fibre with multiple signals propagating through it. So, this section will then treat two phenomenon: Cross Phase Modulation and Four Wave Mixing. Both phenomena are related to the term which is due to third order susceptibility.

\subsubsection{Cross Phase Modulation}

Let's start here with some equations. Let's see we have the electric field which has two frequency components. The first components having an amplitude E1 and a frequency 1 and the second has the amplitude E2 and the frequency 2 and we write the equation:

$$
E=E_{1} e^{j \omega_{1} t}+E_{2} e^{j \omega_{2} t}
$$

If we substitute this into the polarization expression, we will get the nonlinear polarization PNL created inside the material. This polarization multiple nonlinear components: One at $\omega_{1}$, another one at $\omega_{2}$ and other components in the same frequency range as the previous two.

$$
\begin{gathered}
P_{N L}=P_{N L}\left(\omega_{1}\right) e^{j \omega_{1} t}+P_{N L} e^{j \omega_{2} t} \\
\quad+P_{N L}\left(2 \omega_{1}-\omega_{2}\right) e^{j\left(2 \omega_{1}-\omega_{2}\right)}+\ldots \\
P_{N L}\left(\omega_{1}\right)=\chi^{(3)}\left\{\left|E_{1}\right|^{2}+2\left|E_{2}\right|^{2}\right\} E_{1} \\
P_{N L}\left(\omega_{2}\right)=\chi^{(3)}\left\{\left|E_{1}\right|^{2}+2\left|E_{2}\right|^{2}\right\} E_{2} \\
\Delta n_{j} \approx n_{2}\left\{\left|E_{j}\right|^{2}+2\left|E_{3-j}\right|^{2}\right\}
\end{gathered}
$$

With $\mathrm{j}$ being the frequency and $\mathrm{n}_{2}$ the nonlinear coefficient. Note from the delta of the refractive index above, that the nonlinear effects of another signal are twice as higher than those of the signal itself. When the term $|E 3-j|^{2}=0$, the refractive index change will be only dependent on the signal itself as the signal phase in this case gets modified by itself. This change in the refractive index gives a phenomenon that's called the Self Phase Modulation:

$$
\Delta n_{j} \approx n_{2}\left\{\left|E_{j}\right|^{2}\right\}
$$

When that term $|E 3-j|^{2} \neq 0$, this means that the change in the refractive index is not only dependent on the signal itself but due also to other signal that's propagating. The phase then is going to be changed by both the signal as well as the other coexistent signal. This phenomena is called the Cross Phase Modulation (XPM):

$$
\Delta n_{j} \approx n_{2}\left\{\left|E_{j}\right|^{2}+2\left|E_{3-j}\right|^{2}\right\}
$$

In WDM Systems, there will be multiple channel transmissions within the optical fibre and XFM. We know that in WDM Systems, if large number of channels propagate, if there's sufficient power in these channels, there will be change in the phase of each signal due to the existence of the other signals. Also note here, if $n$ channels are transmitting, then, each channel is going to be affected by the power present and remaining in 1-n channels. The collective effect of Cross Phase Modulation is way stronger than what one will see from self-phase modulation.

Now in order to formulate the problem, we can write the field distribution for the $\mathrm{j}$ channel as:

$$
E_{j}=F_{j}(x, y) A_{j}(z) e^{-j \beta_{0 j} z}
$$

Now, if we follow the same steps as were done for the Self Phase Modulation, we'll end up with two equations: One for the field distribution: $\mathrm{Fj}(\mathrm{x}, \mathrm{y})$ and one for the evolution of the envelope: $\mathrm{A}_{\mathrm{j}}$ $(\mathrm{z}) \mathrm{e}-{ }^{\mathrm{j} \beta}{ }_{0}^{\mathrm{jz}}$ is a function of distance on the optical fibre.

Taking the nonlinear variation of the Schrodinger equation, we're talking about the Nonlinear Schrodinger (NLS) equation, an equation belonging to classical field theory [16]. This equation has, among others, applications related to the propagation of light in nonlinear optical fibres [17]. The NLS equation is given as:

$$
\begin{aligned}
\frac{\delta A_{j}}{\delta z}+\beta_{1 j} & \frac{\delta A_{j}}{\delta t}-\frac{j \beta_{2 j}}{2} \frac{\delta^{2} A_{j}}{\delta t^{2}} \\
& +\frac{\alpha_{j}}{2} A_{j}=-j \frac{n_{2} \omega_{j}}{c}\left\{f_{j j}\left|A_{j}\right|^{2}+2 f_{j k}\left|A_{k}\right|^{2}\right\}
\end{aligned}
$$

Where:

$\frac{\delta A_{j}}{\delta z}$ is the rate of the change as a function of distance.

$\beta_{1 j} \frac{\delta A_{j}}{\delta t}$ is the group velocity.

$\frac{j \beta_{2 j}}{2} \frac{\delta^{2} A_{j}}{\delta t^{2}}$ is the group velocity dispersion.

$\frac{\alpha_{j}}{2} A_{j}$ is the attenuation.

$f_{j j}$ is the overlap integral, how much overlap exists between each two signals.

$-j \frac{n_{2} \omega_{j}}{c}\left\{f_{j j}\left|A_{j}\right|^{2}+2 f_{j k}\left|A_{k}\right|^{2}\right\}$ is the nonlinear term.

The overlap integral $f_{j k}$ is given by:

$$
f_{j k}=\frac{\iint\left|F_{j}(x, y)^{2}\right|\left|F_{k}(x, y)^{2}\right| d x d y}{\iint\left|F_{j}(x, y)^{2}\right| d x d y \iint\left|F_{k}(x, y)^{2}\right| d x d y}
$$

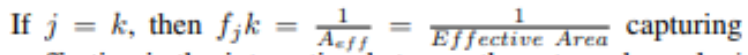
how effective is the interaction between these two channels $j$ and $k$.

The nonlinear Schrödinger (NLS) equation will become:

$$
\begin{aligned}
& \frac{\delta A_{1}}{\delta z}+\frac{1}{v_{g 1}} \frac{\delta A_{1}}{\delta t}-\frac{j \beta_{21}}{2} \frac{\delta^{2} A_{1}}{\delta t^{2}}+\frac{\alpha_{1}}{2} A_{1}=\gamma_{1}\left\{\left|A_{1}\right|^{2}+2\left|A_{2}\right|^{2}\right\} A_{1} \\
& \text { with } \gamma_{1}=\frac{n_{2} \omega_{1}}{c A_{e f f}}
\end{aligned}
$$

In fibre where dispersion is large, there will be sign difference between the velocity of the two signals, and then the walk off time will be very short, so the nonlinear interaction between these two pulses will be very small. Now if one uses the Dispersion Flattened Fibre (DFF), which is a fibre characterized by a smaller mode-field diameter which concentrates optical power in a smaller volume where the increased power density in fibre can cause nonlinear effects [18]. The velocity of the signals going through the DFF will be almost similar, the walk off time will be very long, and the nonlinear interaction between these pulses will be quite significant in a way that will increase the effect of Cross Phase Modulation. 
To avoid Cross Phase Modulation effects in the WDM signal, it is desirable to keep some dispersion into the system. In the WDM system where number of channel is very high, say 128 channels WDM System every channel will have the effect of the remaining 127 channels. Moreover, every channel, even if it just carries the order of 2-3 milliWatts, the total power of 128 channels will be easily in the order of 500 milliWatts, and then the nonlinear phase which will be created because of this will be significant. So, in a WDM System one will see that the Cross Phase Modulation will be much stronger than the Self Phase Modulation because each channel will still carry a power that is very small say 2-3 milliWatt. At such power, the Self Phase Modulation will be very weak, but collectively, the Cross Phase Modulation will be very strong.

In a WDM System if we want channels not to get affected by neighbouring channels due the Cross Phase Modulation, we should get the fibre that has dispersion. You might have read in the literature, that it's desirable to make the dispersion small [19] [20], so that the pulse broadening do not take place. However, now when we are talking about WDM channels, we find that making dispersion 0 is not a good option, as channels then will start interacting and there will be Cross Talk between these channels. So, XPM affects the signals in different frequencies, it also gives the same phenomenon like the spectral broadening which is not symmetrical around the original frequency, you might get asymmetric spectrum developed because of this phenomenon of Cross Phase Modulation. The same phenomenon gives another phenomenon called Four Wave Mixing, which we'll discover in the following section.

\subsubsection{Four Wave Mixing}

Four Wave Mixing in the optical system is similar to inter channel mixing or intermodulation products in the electrical system. When an amplifier goes into saturation, if you put two frequencies inside the amplifier, the third frequency is generated because of the nonlinearities. These two frequencies give the product which is sum and difference of frequencies. The same phenomenon is seen in optical fibres because of the third order susceptibility. If we consider a nonlinear polarization with three signals, or three frequencies simultaneously put in the optical fibre:

$\mathrm{P}_{\mathrm{NL}}=\varepsilon_{0} \chi^{(3)} \vdots \mathrm{E}_{1} \mathrm{E}_{2} \mathrm{E}_{3}$

$$
\begin{gathered}
\omega_{2}=\omega_{1}+2 \Delta \omega \\
\omega_{3}=\omega_{1}+\Delta \omega \\
\omega_{4}=\omega_{1}+\omega_{2}-\omega_{3} \\
=\omega_{1}+\omega_{1}+2 \Delta \omega-\omega_{1}-\Delta \omega \\
=\omega_{1}+\delta \omega \\
=\omega_{3}
\end{gathered}
$$

These three frequencies will produce a fourth frequency $\omega 4=\omega 1$ $\pm \omega 2 \pm \omega 3$ and they will be having the wave numbers:

$\mathrm{k}=\mathrm{k}_{1} \pm \mathrm{k}_{2} \pm \mathrm{k} 3(\mathrm{~A})$

So from three frequencies: $\omega 1, \omega 2, \omega 3$ the following frequencies

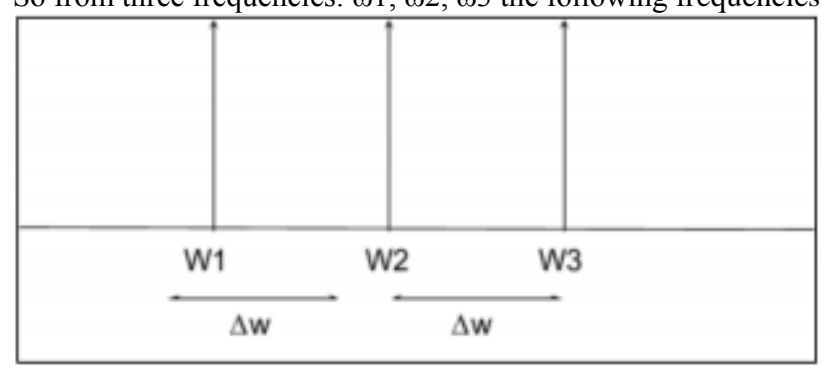

will be generated:

\begin{tabular}{|c|}
$\omega_{1}+\omega_{3}-\omega_{2}$, \\
$\omega_{3}+\omega_{2}-\omega_{1}$, \\
$2 \omega_{1}-\omega_{2}$, \\
$2 \omega_{1}-\omega_{3}$, \\
$2 \omega_{2}-\omega_{3}$, \\
$2 \omega_{2}-\omega_{3}$, \\
$2 \omega_{3}-\omega_{1}$, \\
$2 \omega_{3}-\omega_{2}(\mathrm{~B})$
\end{tabular}

Fig. 3. Equispaced 3 channels in WDM System

From the previous sections, we have defined a formula $\mathrm{P}_{\mathrm{NL}}$ where:

From the above, we see that section A's frequency quantities have the same range or lie within the same frequency band while section B's frequency quantities fall in a different band altogether. For instance, $\omega_{4}$ is almost three times $\omega_{1}$. We will not be looking into those. The phenomenon that frequencies of section B will lie within the same frequency band is what we call Four Wave Mixing, which is also called the inter modulation. This phenomenon is exactly what you see within an amplifier. Let's consider a WDM system where there are 3 channels and as we know in WDM, m wavelengths are equispaced.

This means by this nonlinear process, the two channels $\omega 1$ and $\omega 2$ are going to put power into $\omega 3$. That could be interpreted in terms

$$
\begin{aligned}
P_{N L}=P_{N L}\left(\omega_{1}\right) e^{j \omega_{1} t} & \\
& +P_{N L}\left(\omega_{2}\right) e^{j \omega 2 t} \\
& +P_{N L}\left(2 \omega_{1}-\omega_{2}\right) e^{2 \omega_{1}-\omega_{2}} \\
& +\cdots
\end{aligned}
$$

With:

$$
\begin{aligned}
& P_{N L}\left(\omega_{1}\right)=\chi^{(3)}\left\{\left|E_{1}\right|^{2}+2\left|E_{2}\right|^{2}\right\} E_{1} \\
& P_{N L}\left(\omega_{2}\right)=\chi^{(3)}\left\{2\left|E_{1}\right|^{2}+\left|E_{2}\right|^{2}\right\} E_{2} \\
& \Delta n_{j} \approx n_{2}\left\{\left|E_{j}\right|^{2}+2\left|E_{3-j}\right|^{2}\right\}
\end{aligned}
$$

of Cross Talk between $\omega 1$ and $\omega 3$ and $\omega 2$ and $\omega 3$. There will be a Cross Talk phenomenon between all WDM Channels. To avoid this phenomenon, it looks like we shouldn't propagate channels that are equispaced. Making dispersion 0 on optical fibre is not a good option. We should rather be keeping some residual dispersion to reduce the nonlinear effects in a multichannel transmission.

As a side note, it's worth mentioning that the phenomenon of Four Wave Mixing is not always considered undesirable. It is actually quite beneficial for wavelength conversion. In paper [21], the authors have shown that using a length of $1 \mathrm{~mm}$ in Semiconductor Optical Amplifiers (SOAs) improves the efficiency by $20 \mathrm{~dB}$ with respect to standard devices, which is $0.5 \mathrm{~mm}$ long.

\subsubsection{Simulations}

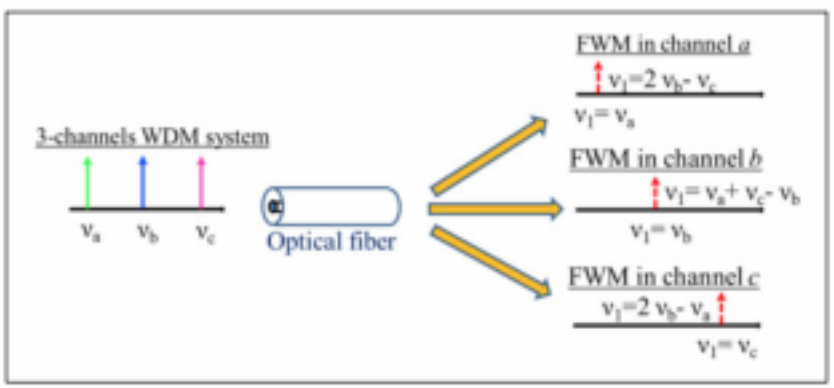

Fig. 1. FWM in a 3 Channels WDM System

The graph in figure 3 represents the number of mixing product terms (M) as a function of number of wavelength where:

$$
M=\frac{N^{2}(N-1)}{2}
$$


As you have seen from the previous section, for a total of 3 input wavelengths, we've obtained a total of 9 mixing product terms.

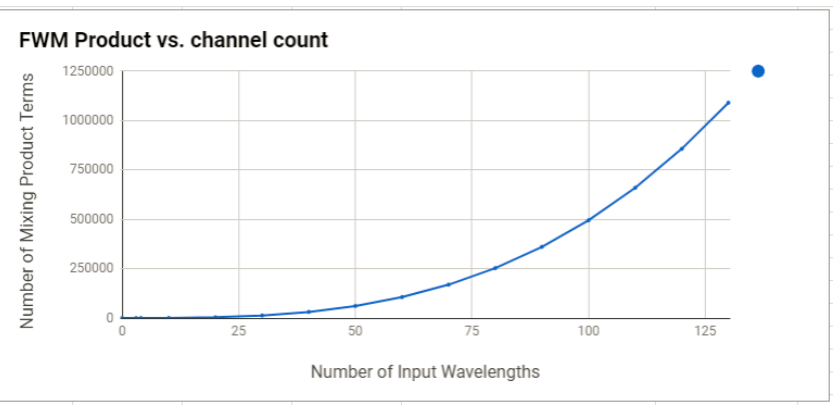

Fig. 4. FWM Product vs. Channel Count

Figure 4 represents how this product is changing as the number of input wavelengths increases. As you can see on the graph, for an input of 10 wavelengths, we obtain 450 mixing product terms. If we triple the number of input wavelengths to 30 , this results in 13050 total mixing products. That is 2900 times higher. This is almost a turning point in the graph. Post that, we see that the FWM product continue to increase very rapidly reaching $1,090,050$ terms for only 130 input wavelengths.

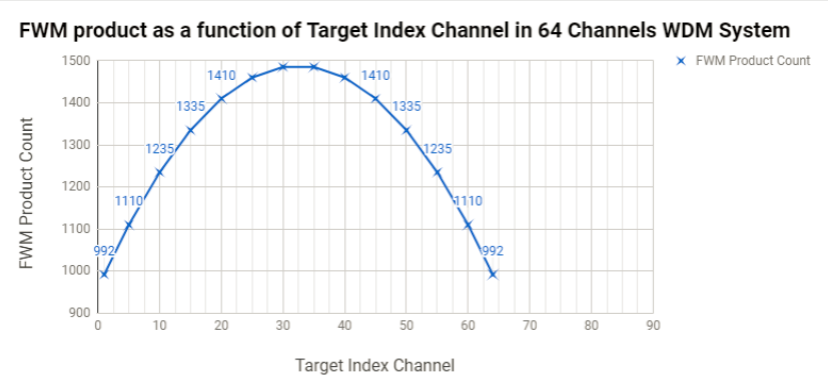

Fig. 5. FWM product as a function of Target Index Channel in 64 Channels WDM System

The next graph in Figure 5 depicts Four Wave Mixing as a function of the Target Index Channel in 64 Channels WDM System. In the case considered with 64 subcarriers, the 33th target index (subcarrier) plays a role in around 1485 FWM combinations while the 1st and the 64th subcarriers have contribution to only approximately 1000 FWM combinations.

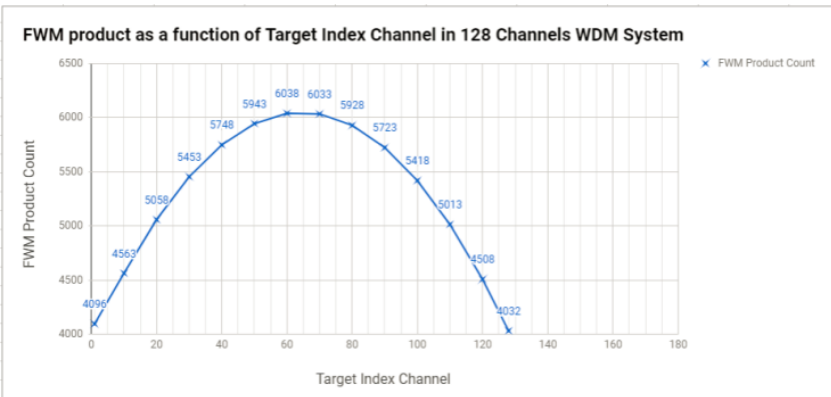

Fig. 6. FWM product as a function of Target Index Channel in 128 Channels WDM System

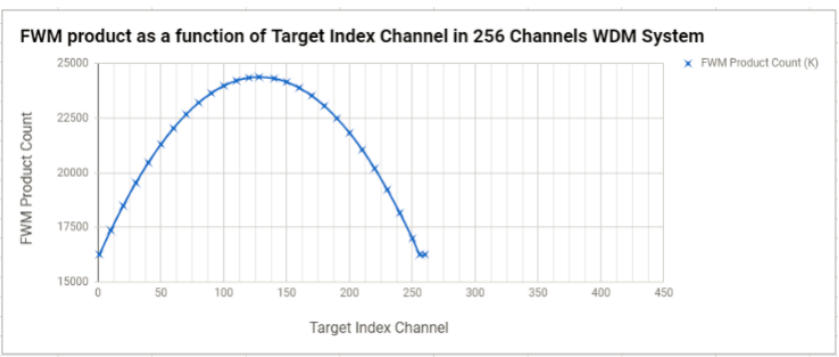

Fig. 7. FWM product as a function of Target Index Channel in 256 Channels WDM System
Figures 6, and 7 represent the same principle but for cases of more subcarriers: 128 , and 256 consecutively.

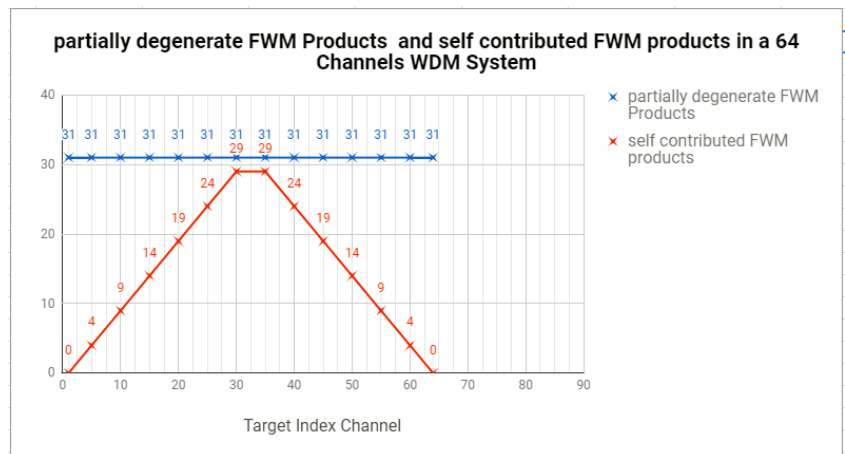

Fig. 8. Partially degenerate FWM Products and self-contributed FWM products in a 64 Channels WDM System

If we look at the next graph in Figure 8, we can see a representation of the partially degenerate FWM products in a 64 Channels WDM System. Before explaining what's on the graph, a quick recall of the theory is necessary. Four Wave Mixing, is known as a 3 rd order nonlinear process where 3 frequencies $\left(\omega_{\text {pump-1 }}, \omega_{\text {pump-2 }}\right.$, and $\left.\omega_{\text {signal }}\right)$ mix in a nonlinear medium, resulting in 2 extra frequencies (a lower frequency $\omega_{\text {Stokes}}$, and a higher frequency $\left.\omega_{\text {antistokes }}\right)$ [22] [23]. When $\omega$ pump $-1=\omega$ pump $-2=\omega_{\text {pump }}$, the process is referred to as Partially Degenerate Four Wave Mixing (PDFWM). We can see from the graph that the degenerate products number is constant within the subcarrier band, meaning that these subcarriers are affected in an equal fashion. The same principle applies when we studied WDM Systems of 128 and 256 Channels, as illustrated in Figure 9, and 10.

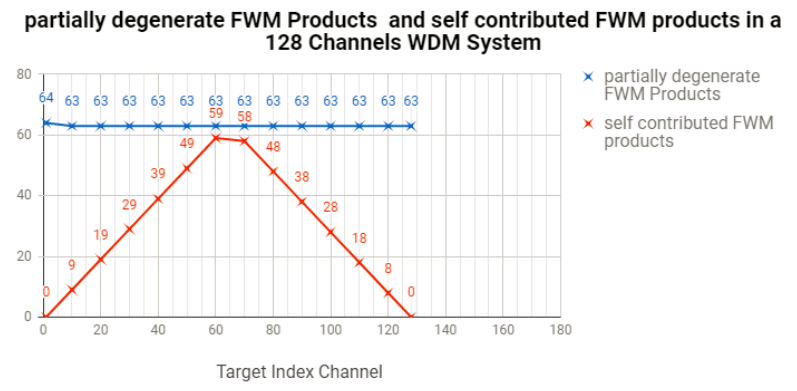

Fig. 9. Partially degenerate FWM Products and self-contributed FWM products in a 128 Channels WDM System

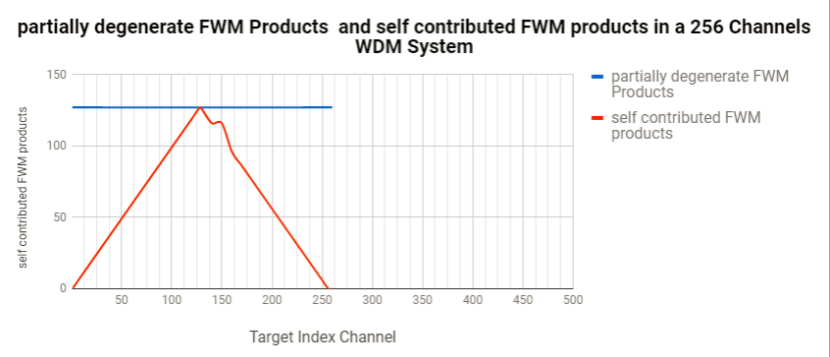

Fig. 10. Partially degenerate FWM Products and self-contributed FWM products in a 256 Channels WDM System

\section{Conclusion}

In summary, we've begun analysing nonlinear effects in bulk material vs. fibre and found that we've seen that high power is required so that nonlinearities can start getting induced effectively and allow researchers to study their effects. Specifically, you can see in bulk material with 1 Watt of power, the same effect inside optical fibre can be seen with only 1 nano Watt of power. We then moved to study the propagation of light within the optical fibre 
and saw how very pronounced inside the optical fibre they are, as a result of the very large interaction length inside the optical fibre. We've also seen how fibre dispersion has little effect on XPM, while a big negative impact on FWM, while more of it results in rather less SPM. The next sections highlighted the analytical model of these nonlinear effects as well as their simulations following the same pattern as we've kept testing with increasing number of channels within the WDM System.

\section{References}

[1] M. Fox, Optical Properties of Solids, ser. Oxford Master Series in Physics. OUP Oxford, 2010. [Online]. Available: https://books.google.ie/books?id=5WkVDAAAQBAJ

[2] "Heinrich hertz's wireless experiment (1887)," 2017. [Online] Available: http://people.seas.harvard.edu/ jones/cscie129/nu lectures/ lecture6/hertz/Hertz exp.html

[3] J. C. Maxwell, "A dynamical theory of the electromagnetic field," Philosophical Transactions of the Royal Society of London, vol. 155 , no. 0, pp. 459-512, jan 1865. [Online]. Available: https://doi.org/10.1098/rstl.1865.0008

[4] R. Ramaswami, K. Sivarajan, and G. Sasaki, Optical Networks: A Practical Perspective, 3rd Edition. Morgan Kaufmann, 2009. [Online]. Available: https://www.amazon.com/OpticalNetworksPractical-Perspective-3rd/dp/0123740924?SubscriptionId= 0JYN1NVW651 KCA56C102\&tag=techkie-20\&linkCode $=x m 2 \&$ camp $=2025 \&$ creative $=165953 \&$ creative $A S I N=0123740924$

[5] R. Ramaswami and K. Sivarajan, Optical Networks: A Practical Perspective (Morgan Kaufmann Series in Networking). Morgan Kaufmann, 2001. [Online]. Available: https://www.amazon.com/Optical-NetworksPractical-PerspectiveNetworking/dp/1558606556?SubscriptionId= 0JYN1NVW651 KCA56C102\&tag=techkie-20\&linkCode $=x \mathrm{x} 2 \&$ camp $=2025 \&$ creative $=165953 \&$ creative $A S I N=1558606556$

[6] G. Agrawal, Nonlinear Fiber Optics, Second Edition (Optics and Photonics). Academic Press, 1995. [Online]. Available: https://www.amazon.com/NonlinearFiber-Optics-SecondPhotonics/dp/0120451425?SubscriptionId= 0JYN1NVW651 KCA56C102\&tag=techkie-20\&linkCode $=x m 2 \&$ camp $=2025 \&$ creative $=165953 \&$ creative $A S I N=0120451425$

[7] T. B. Benjamin and J. E. Feir, "The disintegration of wave trains on deep water part 1. theory," Journal of Fluid Mechanics, vol. 27, no. 03, p. 417, feb 1967. [Online]. Available: https: //doi.org/10.1017/s002211206700045x

[8] T. B. Benjamin and K. Hasselmann, "Instability of periodic wavetrains in nonlinear dispersive systems [and discussion]," Proceedings of the Royal Society A: Mathematical, Physical and Engineering Sciences, vol. 299, no. 1456, pp. 59-76, jun 1967. [Online]. Available: https://doi.org/10.1098/rspa.1967.0123

[9] S. Obayya, Computational Photonics. Imprint unknown, 2011. [Online].

Available: https://www.amazon.com/ComputationalPhotonics-SalahObayya/dp/1119957508?SubscriptionId=

0JYN1NVW651 KCA56C102\&tag=techkie-20\&linkCode $=x m 2 \&$ camp $=2025 \&$ creative $=165953 \&$ creative ASIN $=1119957508$

[10] L. R. D. Sam-Shajing Sun, Introduction to Organic Electronic and Optoelectronic Materials and Devices (Optical Science and Engineering). CRC Press, 2008. [Online]. Available: https://www.amazon.com/Introduction-ElectronicOptoelectronicMaterials-Engineering/dp/0849392845?SubscriptionId= 0JYN1NVW651 KCA56C102\&tag=techkie-20\&linkCode $=x m 2 \&$ camp $=2025 \&$ creative $=165953 \&$ creative $A S I N=0849392845$

[11] C. Li, Nonlinear Optics: Principles and Applications. Springer, aug 2016. [Online]. Available: https://www.xarg.org/ref/a/9811014876/

[12] P. R. Shevgaonkar. (2012, jan) Introduction to non-linear fiber optics. [Online]. Available: https://goo.gl/2MMaqD

[13] S. Dhara, F. Araoka, M. Lee, K. V. Le, L. Guo, B. K. Sadashiva, K. Song, K. Ishikawa, and H. Takezoe, "Kerr constant and third-order nonlinear optic susceptibility measurements in a liquid crystal composed of bent-shaped molecules," Physical Review E, vol. 78, no. 5, nov 2008.2 [Online]. Available: https://doi.org/10.1103/physreve.78.050701

[14] D. B. Potter. (2010, aug) Module 3 - attenuation in optical fibers [Online]. Available: http://opti500.cian-erc.org/opti500/pdf/sm/ Module3\%200ptical\%20Attenuation.pdf

[15] R. W. Boyd. (2008) Nonlinear optics, third edition. [Online]. Available: https://www.amazon.com/Nonlinear-Optics-ThirdRobert-
Boyd/dp/0123694701?SubscriptionId=0JYN1NVW651KCA56C10 $2 \&$ tag=techkie-

20\&linkCode $=x m 2 \&$ camp $=2025 \&$ creative $=165953 \& \quad$ creativeASIN $=0123694701$

[16] S. A. Rashkovskiy. (2018, feb) Nonlinear schrodinger equation and classical-field description of the lamb retherford experiment [Online]. Available: https://arxiv.org/pdf/1802.01979.pdf

[17] D. Felice, "A study of a nonlinear schrodinger equation for optical " fibers," Ph.D. dissertation, Facolta di Scienze Matematiche, Fisiche e`Naturali, 2016.

[18] F. C. F. O. F. S. Co.). (2016, nov) Optical fiber dispersion. [Online]. Available:

https://www.fiberoptics4sale.com/blogs/archiveposts/95047942optical-fiber-dispersion

[19] J. D. Downie, J. Hurley, and X. Zhu, "Xpm and sbs nonlinear effects on mlse with varying uncompensated dispersion," Opt. Express, vol. 17 , no. 24 , pp. 22 240-22 245, Nov 2009. [Online] Available: http://www.opticsexpress.org/abstract.cfm?URI=oe-1724-22240

[20] M. Takahashi, R. Sugizaki, J. Hiroishi, M. Tadakuma, Y. Taniguchi, and T. Yagi, "Low-loss and low-dispersion-slope highly nonlinear fibers," J. Lightwave Technol., vol. 23, no. 11, p. 3615, Nov 2005. [Online]. Available: http://jlt.osa.org/abstract.cfm?URI=jlt-23-113615

[21] A. D’Ottavi, F. Girardin, L. Graziani, F. Martelli, P. Spano, A. Mecozzi, S. Scotti, R. Dall'Ara, J. Eckner, and G. Guekos, "Fourwave mixing in semiconductor optical amplifiers: a practical tool for wavelength conversion," IEEE Journal of Selected Topics in Quantum Electronics, vol. 3, no. 2, pp. 522-528, Apr 1997.

[22] T. N. H. D. V. Aravind P. Anthur, Regan T. Watts and L. P. Barry. (2013, aug) A general phase noise relationship for four-wave mixing. [Online]. Available: https://arxiv.org/pdf/1308.0914.pdf

[23] S. K. O. R. I. L. Y. N. I. Motoki Asano, Yuki Takeuchi and T. Yamamoto. (2016, may) Stimulated brillouin scattering and brillouin-coupled four-wave-mixing in a silica microbottle resonator. [Online]. Available: https://arxiv.org/pdf/1605.07287.pdf 